\title{
An Objective Assessment Scale for "Come-to-Sit" Using a Specifically Designed Jacket in Stroke Patients
}

\author{
Jun Ho Lee, M.D., Han Young Jung, M.D., Jae Woo Lee, Ph.D. ', Kyung Lim Joa, M.D., Jae Hong Kim, M.D., \\ Myung Jong Kim, RPT, Do Hang Hur², Eun Ju Jang ${ }^{2}$, Myeong Ok Kim, M.D.
}

Department of Physical \& Rehabilitation Medicine, Inha University School of Medicine,

${ }^{1}$ Department of Physics, Inha University and ${ }^{2}$ Technical Aid Center, Notre-dame Welfare Center, Incheon 400-711, Korea

Objective To compare an objective assessment scale for "come-to-sit" in stroke patients with the previously established subjective assessment scales of "performance-based assessment" and the "ability for basic movement scale".

Method A specifically designed jacket was used to determine the objective degree of assistance needed for patients to perform the task. While patients were sitting up, the investigator evaluated the amount of assistance needed in a fully dependent state (A) and with maximal effort (B). Using this measure, we obtained an objective scale, $\{(A-B) / A\} \times 100$. In addition, patients were tested in two starting positions: hemiplegic-side lying and soundside lying. We then compared the objective scale with subjective scales and other parameters related to functional outcomes.

Results For both starting positions, the objective assessment scale showed high correlation with the previously established subjective scales $(\mathrm{p}<0.01)$. Only the hemiplegic-side lying-to-sit objective scale showed a significant correlation with the parameters used to assess functional outcomes $(\mathrm{p}<0.05)$. In terms of Brunnström stages, only the leg stage showed a significant correlation with the objective "come-to-sit" scale $(\mathrm{p}<0.01)$.

Conclusion The objective scale was comparable to established subjective assessment scales when used by an expert. The hemiplegic-side lying-to-sit maneuver had a high correlation with patient's functional recovery. Specifically, balance and lower extremity function appear to be important factors in the "come-to-sit" activity.

Key Words Come-to-sit, Objective assessment scale, Assistance jacket

Received June 15, 2009; Accepted September 1, 2010

Corresponding author: Myeong Ok Kim

Department of Physical \& Rehabilitation Medicine, Inha University

Hospital, 7-206, 3-Ga, Shinheung-dong, Jung-gu, Incheon 400-711, Korea.

Tel: +82-32-890-2480, Fax: +82-32-890-2486, E-mail: rmkmo@inha.ac.kr (c) This is an open-access article distributed under the terms of the Creative Commons Attribution Non-Commercial License (http:// creativecommons.org/licenses/by-nc/3.0) which permits unrestricted noncommercial use, distribution, and reproduction in any medium provided the original work is properly cited.

Copyright ( 2012 by Korean Academy of Rehabilitation Medicine

\section{INTRODUCTION}

Although the position changing maneuver known as "come-to-sit" is routine and performed several times a day, it is one that may present performance challenges after central nervous system (CNS) injury. A person who is unable to take a sitting posture will encounter limitations in activities of daily living (ADL) and will 
also be problematic for care-givers. ${ }^{1,2}$ Since the main goal of rehabilitation is to diminish dependency when performing ADLs, retraining of the "come-to-sit" activity is necessary to achieve ADL independence. ${ }^{3}$

In rehabilitation of CNS injury, the movement between a lying and sitting posture is an essential part of the recovery phase. In order to accomplish tasks such as feeding, washing of hands, grooming and dressing, the patient must achieve a sitting posture, after which advances into transfer activity and gait are possible. In stroke patients, the development of "come-to-sit" activity is divided into three stages: a total assist requiring state, the decreasing of assist, and an independent state. Susan and Kathryn ${ }^{4}$ analyzed and confirmed this pattern examining selective patterns of trunk movement from a side lying to a sitting posture. Yet assessment of this task has mostly depended on subjective tools such as 'performance-based assessment (PBA) ${ }^{5}$ or 'the ability for basic movement scale' (ABMS). ${ }^{6}$

This study was therefore designed to resolve the following issues: first, to devise an objective assessment for "come-to-sit" maneuvers, using quantitative analysis with a specifically designed jacket and a measurement scale. Second, to find the correlation between the subjective assessment tools (PBA or ABMS) and assessment of the side lying posture, for both hemiplegicside and sound-side lying. Third, to investigate the correlation between the subjective analysis of acquired independence on "come-to-sit" and "performancebased, ability to basic movement scales' such as the Korean version of the Berg scale (K-BBS), ${ }^{7}$ the Korean modified Barthel index (K-MBI), ${ }^{8}$ the Korean mini-mental status examination (K-MMSE), ${ }^{9}$ and the Brunnström stage. $^{10}$

\section{MATERIALS AND METHODS}

\section{Subjects}

Twenty two outpatients (14 male, 8 female) with stroke who visited rehabilitation between December 2008 and May in 2009 were enrolled. The mean age was 62.5 years. The subject group consisted of 15 patients with cerebral infarction and 7 with intracranial hemorrhage. 12 patients had left hemiplegia, and 21 were right handed. The average time from injury was $198.5 \pm 318.2$ days and subjects' average body weight was $60.2 \pm 11.9$ kilograms.
Patients who were unable to obey commands or had limitations in their range of movement were excluded.

\section{Methods}

All participants in the PBA and ABMS were tested by same examiner, who was an expert practitioner in the field. Based on PBA $^{5}$ scales, patients were subdivided into 5 stages: independence, supervision, minimal assistance, moderate assistance and maximal assistance.

Independence (stage 1) was defined as the 'patient is able consistently to perform the skill safely without no one present,' whereas supervision (stage 2) denoted that the 'patient requires someone within arm's reach as a precaution'. Minimal assistance (stage 3 ) meant that the 'patient is able to complete a majority of the activity without assistance.' 'The patient is able to complete part of the activity without assistance' and 'the patient is unable to assist in any part of the activity' were defined as fourth and fifth stages, respectively (Appendix 1). ABMS scores were organized into five stages based on sitting ability, defined as follows. ${ }^{6}$ Stage 0 denotes a prohibition from moving because of a medical condition. Stage 1 is a totally dependent stage that requires more than $75 \%$ assistance. Stage 2 is partially dependent stage, with a $<75 \%$ assistance requirement. Stage 3 is the 'independent in special environments' stage, wherein movement by holding a handrail or sitting is possible, and stage 4 is a



Fig. 1. The specifically designed jacket was applied to pull the patient's trunk so as to measure the weight. 
completely independent stage, wherein sitting is possible without assistance (Appendix 2).

We created a specially designed jacket (Fig. 1) with a hook attached to two scales (Travelon ${ }^{\circledR}$, Elk Grove Village, USA) (Fig. 2) measuring the maximal value of pulling weight, so that the patient could be pulled in the same direction while sitting constantly with repetitive assessments. The specially designed jacket was tailored in its vest shape by an experienced orthotist and tailored for comfortable fit (Fig. 1). To maintain a constant angle as the patient went from a supine to sitting position, we attached several hooks at the front of jacket to connect the two scales. Through a process of investigation, we determined the best position for the hooks to pull the weight with even distribution.

A patient wearing the jacket was ordered to move into

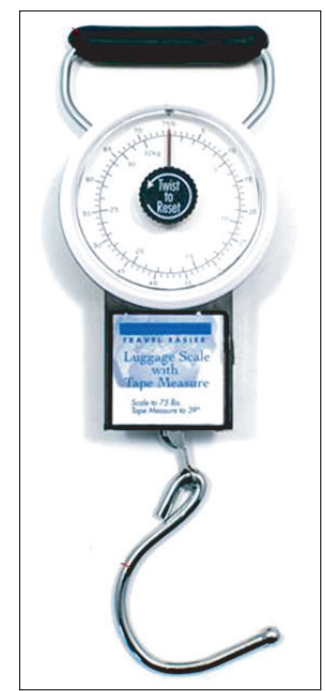

Fig. 2. Scale measuring the maximal value of pulling weight (Travelon $^{\circledR}$, Elk Grove Village, USA). "come-to-sit" from a side lying posture, and the examiner supported the patient's buttock with a knee while assisting the patient by pulling the attached scales with both hands. The test was divided into patient with total dependency (Fig. 3-A) and maximal effort to take "cometo-sit" posture independently (Fig. 3-B). The maximum weight loaded on the scale during taking the "come-tosit" posture was measured three times, then calculated on the side lying to sit objective scale (SSOS) using the following equation.

$$
\text { SSOS }=\frac{A-B}{A} \times 100(\%)
$$

Before proceeding, we performed a pilot study to verify the reliability of the SSOS measurement tool. The pilot study was to determine reliabilities between examiners and within the same examiner's results. The interrater reliabilities among examiners were analyzed by Spearman correlation coefficient (rho) with 4 examiners who used the SSOS with 10 patients. The intra-rater reliabilities for individual examiners were also analyzed by Spearman correlation coefficient, with an examiner performing SSOS measurements four times for 10 patients within 12 hours. The result of this pilot study for the inter-rater reliability among examiners (r, correlation coefficient) was $0.989-0.997(\mathrm{p}<0.01)$ and the intra-rater reliability ( $\mathrm{r}$ ) within examiners was 0.997-1.000 ( $\mathrm{p}<0.01)$. These results indicated that the SSOS measurement was highly reliable (Table 1).

\section{Statistical analysis}

The movement from side lying to the "come-tosit" posture was assessed on the sound side and the hemiplegic side, and the PBA, ABMS, SSOS values for these sides were compared through nonparametric
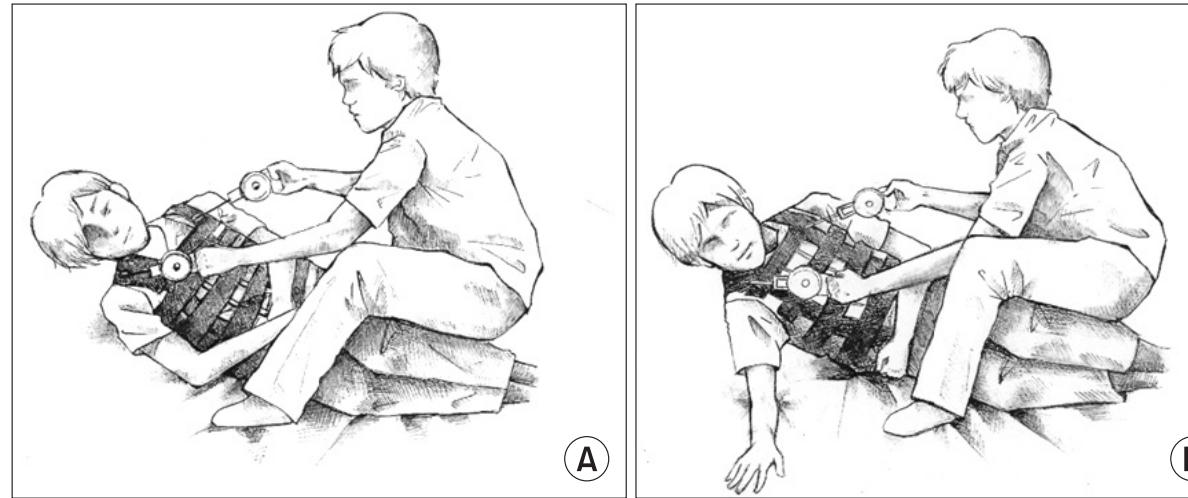

Fig. 3. (A) Total assisted sitting with full dependence. (B) Active assisted sitting with full effort. 
analysis. The calculated objective values for the K-BBS, K-MBI, K-MMSE and for the Brunnström stages of hand, upper and lower limb were also analyzed by the Spearman correlation coefficient.

\section{RESULTS}

Comparison of the values measured from taking the "come-to-sit" posture from a side lying posture on the hemiplegic side or sound side

The PBA values for hemiplegic side lying to sitting were as follows: 2 patients were at stage 1,4 at stage 2,4 at stage 3 , and 7 at stages 4 and 5 . The PBA values for sound side lying to sitting were $1,1,2,1$ and 17 patients at the respective stages 1-5. ABMS scores for the hemiplegic side were: 1 patient at stage 0,5 at stage 1,5 at stage 2 ,

Table 1. Interrater and Intrarater Reliability for the Side lying to Sit Objective Scale

\begin{tabular}{llll}
\hline \multicolumn{2}{c}{ Interrater reliability } & \multicolumn{2}{c}{ Intrarater reliability } \\
\hline Tester $1-2$ & $\mathrm{r}=0.995^{*}$ & Test day $1-2$ & $\mathrm{r}=0.999^{*}$ \\
Tester $1-3$ & $\mathrm{r}=0.989^{*}$ & Test day $1-3$ & $\mathrm{r}=1.000^{*}$ \\
Tester $1-4$ & $\mathrm{r}=0.996^{*}$ & Test day $1-4$ & $\mathrm{r}=0.998^{*}$ \\
Tester $2-3$ & $\mathrm{r}=0.992^{*}$ & Test day $2-3$ & $\mathrm{r}=0.999^{*}$ \\
Tester $2-4$ & $\mathrm{r}=0.997^{*}$ & Test day $2-4$ & $\mathrm{r}=0.997^{*}$ \\
Tester $3-4$ & $\mathrm{r}=0.993^{*}$ & Test day $3-4$ & $\mathrm{r}=0.998^{*}$ \\
\hline
\end{tabular}

r: Correlation coefficient

${ }^{*} \mathrm{p}<0.05$

(A)

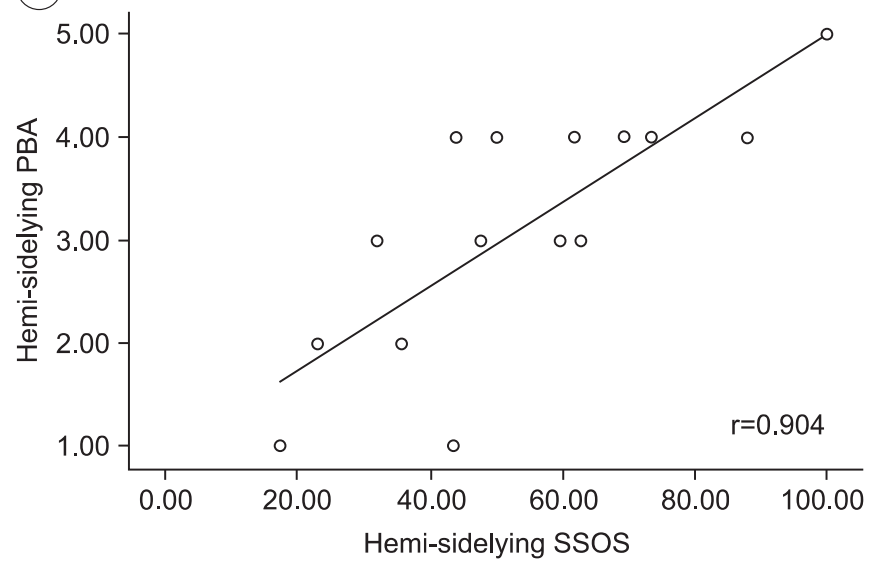

6 at stage 3 , and 5 at stage 4 . ABMS scores for the sound side were $1,0,3,2$, and 16 patients, respectively. Since SSOS was measured as $60.9 \%( \pm 28.0)$ when sitting on the hemiplegic side and $89.0 \%( \pm 23.2)$ when sitting on sound side, these results indicated that lying on the sound side made it easier for patient to take the "cometo-sit" posture. The hemiplegic and sound side values were compared through nonparametric analysis, and all of the PBA, ABMS, SSOS categories showed statistically significant differences $(\mathrm{p}<0.01)$. There was no statistical difference between cerebral hemorrhage and cerebral infarction group.

Correlation between SSOS values and subjective assessment tools for hemiplegic side lying

The Spearman nonparametric correlation coefficient analyses between the values of SSOS, PBA and ABMS for the hemiplegic side lying to sit position were as follows: SSOS and PBA $(r=0.904, p<0.01)$ and SSOS and ABMS $(r=0.880, p<0.01)$, showing statistically significant correlations (Fig. 4).

\section{Correlation between SSOS values and subjective assessment tools for sound side lying}

The Spearman nonparametric correlation analyses between the SSOS, PBA and ABMS for the sound side lying to sit position were as follows: SSOS and PBA $(\mathrm{r}=0.850, \mathrm{p}<0.01)$, SSOS and ABMS $(\mathrm{r}=0.767, \mathrm{p}<0.01)$.

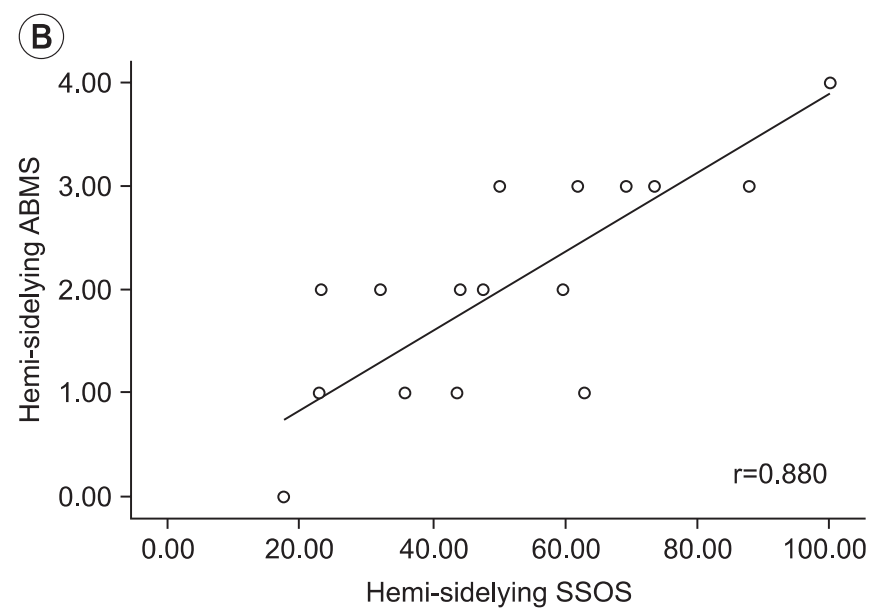

Fig. 4. (A) The correlations between SSOS and PBA at the hemiplegic side. (B) The correlations between SSOS and ABMS at the hemiplegic side. SSOS: Side lying to sit objective scale, PBA: Performance-based assessment, ABMS: The ability for basic movement scale. 
(A)

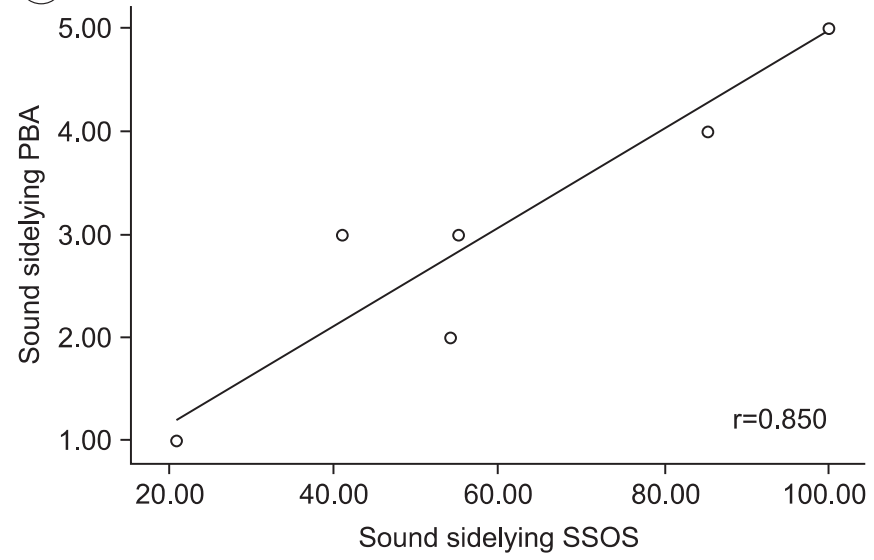

(B)

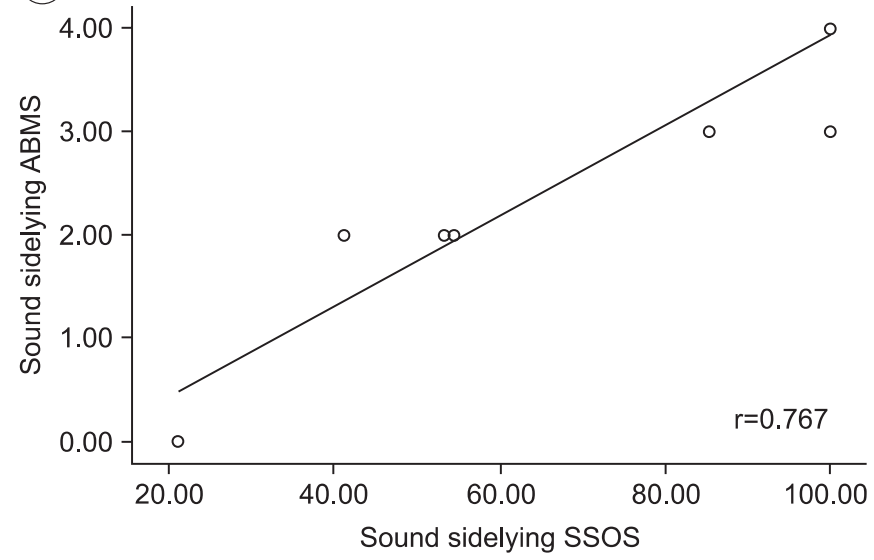

Fig. 5. (A) The correlations between SSOS and PBA at the sound side. (B) The correlations between SSOS and ABMS at the sound side. SSOS: Side lying to sit objective scale, PBA: Performance-based assessment, ABMS: The ability for basic movement scale.

Table 2. The Relationship between SSOS and Parameters Measuring Functional Outcomes for Hemiplegic and Sound Sides

\begin{tabular}{lcc}
\hline & $\begin{array}{c}\text { SSOS at } \\
\text { hemiplegic side } \\
\text { correlation } \\
\text { coefficient (r) }\end{array}$ & $\begin{array}{c}\text { SSOS at sound side } \\
\text { correlation } \\
\text { coefficient (r) }\end{array}$ \\
\hline Brunnstrom hand & 0.285 & -0.193 \\
Brunnstrom arm & 0.340 & -0.069 \\
Brunnstrom leg & $0.682^{*}$ & 0.357 \\
K-MMSE & $0.634^{*}$ & 0.414 \\
K-MBI & 0.488 & 0.425 \\
K-BBS & $0.607^{*}$ & 0.385 \\
\hline
\end{tabular}

SSOS: Side lying to sit objective scale, K-MMSE: Korean version of mini-mental status examination, K-MBI: Korean version of modified Barthel index, K-BBS: Korean version of the Berg balance scale ${ }^{*} \mathrm{p}<0.05$

These reflect statistically significant correlations (Fig. 5).

Correlation between sSos values and functional assessment tools for both hemiplegic and sound side lying

The SSOS for hemiplegic side lying to sit showed statistically significant correlations with the Brunnström lower limb stage, the K-BBS, and the K-MMSE when using Spearman nonparametric correlation coefficient analysis $(\mathrm{p}<0.05)$, but there were no significant correlations with the Brunnström stages of hand and upper limb, or the K-MBI ( $p>0.05$ ) (Table 2). The SSOS for sound side lying to sit revealed no statistical correlations with any of the examined functional assessment tools.

\section{DISCUSSION}

The "Come-to-sit" transfer maneuver is one of the most important basic actions in activities of daily living. If a child with cerebral palsy can sit alone before 2 years of age, the chance of his acquiring independent ambulation is very high. However, if the child is not able to sit alone by the age of 4 , he is unlikely walk independently. ${ }^{11}$ This finding suggests that independent sitting ability has great influence on future activities of daily living.

The reliability of subjective assessment tools rarely surpass those of objective assessment tools, but there has not been sufficient data relating to the previously utilized assessment tools for sitting maneuvers. Quantitative analysis of sitting maneuver ability has never been attempted. This study therefore has significance because it is the first to use an objective assessment tool to substantiate the reliabilities of 5 stage measuring methods based on clinical decision through observation. We invented a new, specially-designed jacket which can constantly measure the level of assistance needed for "come-to-sit". To determine the proper position at which to attach the scale to enable constant weight 
measurement during "come-to-sit", we analyzed the force of pulling scale, with the aid of physicists. The success of this was proven by the reliability analysis between examiners and within examiners for the assessment tool.

In general, the "come-to-sit" transfer maneuver has three trunk initiation patterns. First, the upper trunk is rotated to the left side due to rotation started from the upper trunk and body weight supported by extension of the upper limb then the forearm. The right upper limb extends furthermore and the left upper limb flexes more with the left hip and thigh supporting the body weight in lower limbs. The second pattern starts in the lateral lower trunk and the extended arm pattern corresponds with the lower trunk lateral flexion to the left side, the convex to the left side, with weight supported on the unilateral extended arm and body weight supported with the left hip and thigh. Third, the flexed arm pattern starts with the upper trunk in a lateral position, puts the upper trunk in a left lateral flexion position with left convexity, and weight is supported with the unilateral left forearm then with hip and thigh. ${ }^{4}$

Using quantitatively objective analysis of the "cometo-sit" posture, subjective assessment conducted by an experienced examiner had highly significant correlation with objective assessment in this study. Therefore, it was indicated that subjective assessment by an experienced examiner is suitable for clinical use. However, if examiners are not experienced as those in this study, assessment errors during evaluation could be problematic. Therefore, the development of standardized tools for quantitative measurement, such as the jacket developed in this study, is necessary.

Hemiplegic patients usually depend on their sound side arm to perform the "come-to-sit" maneuver. At present, subjective assessment tools for evaluating the "cometo-sit" maneuver have tended to evaluate the side with less difficulty with regardless of hemiplegic or sound side. We noticed that assessment using the hemiplegic side has more significant correlation with the patient's recovery of functional outcomes in this study. However, this result was not meant to encourage patients to sit on their hemiplegic side, and evaluation of the assistance requirement during the "come-to-sit" maneuver on the hemiplegic side had a more objective relationship with the prediction of patient's recovery.

SSOS values for hemiplegic side sitting had no signifi- cant correlation with values related to activities of daily living such as the K-MBI, but the Brunnström lower limb stage and the K-BBS showed statistically significant correlation with SSOS values for the hemiplegic side. Based on this correlation, we could assume that a sense of balance and the functional recovery of the lower limb play an important role in performing the "cometo-sit" posture. However, given that there were only 22 participants enrolled in this study, more participants would be needed to generalize these results.

\section{CONCLUSION}

By quantization of stroke patient's "come-to-sit" maneuver using a specifically designed jacket and scales, this study is significant in that it is the first to evaluate the reliability of pre-existing subjective assessment tools. It found that assessment of the hemiplegic side lying to sit maneuver has more advantages for predicting the progression of patient's functional recovery over the routinely performing assessment of the sound side lying to sit maneuver.

\section{ACKNOWLEDGEMENTS}

This study was supported by a grant from Inha University.

\section{REFERENCES}

1. Chon JS, Chun SI, Kim DA, Seo CH, Yun TJ, Jang YW. The analysis of sit-to-stand motion in stroke patient. J Korean Acad Rehab Med 2000; 24: 850-856

2. Kim MG, Kim SH, Yang SH, Son SG. An analysis of the movements during rising from a chair. J Korean Acad Rehab Med 1995; 19: 23-31

3. Hsieh CL, Sheu CF, Hsueh IP, Wang CH. Trunk control as an early predictor of comprehensive activities of daily living function in stroke patients. Stroke 2002; 33: 2626-2630

4. Susan RY, Kathryn LE. Functional movement reeducation, 1st ed, New York: Churchill Livingstone, 1997, 375-396

5. Susan BO. Physical rehabilitation assessment and treatment, 3rd ed, Philadelphia: F.A. Davis Company, 1994, 196-197 
6. Hashimoto K, Hiquchi K, Nakayama Y, Abo M. Ability for basic movement as an early predictor of functioning related to activities of daily living in stroke patients. Neurorehabil Neural Repair 2007; 21: 353357

7. Jung HY, Park JH, Shim JJ, Kim MJ, Hwang MR, Kim SH. Reliability test of Korean version of berg balance scale. J Korean Acad Rehab Med 2006; 30: 611-618

8. Jung HY, Park BK, Shin HS, Kang YK, Pyun SB, Nam JP, Kim SH, Kim TH, Han TR. Development of the Korean version of modified barthel index (K-MBI): multicenter study for subjects with stroke. J Korean Acad Rehab Med 2007; 31: 283-297
9. Jhoo JH, Kim KW, Lee DY, Youn JC, Lee TJ, Choo IH, Ko HJ, Seo EH, Woo JI. Comparison of the performance in two different Korean versions of minimental status examination: MMSE-KC and K-MMSE. J Korean Neuropsychiatr Assoc 2005; 44: 98-104

10. Brandstater ME. Stroke rehabilitation. In: Delisa JA, Editor. Physical medicine and rehabilitation, 4th ed, Philadelpia: Lippincott Williams \& Wilkins, 2005, 1667-1668

11. Molnar GE, Gordon SU. Cerebral palsy: predictive value of selected clinical signs of early prognostication of motor function. Arch Phys Med Rehabil 1976; 57: 153-158 
Appendix 1. Performance-based Assessment (PBA) ${ }^{5}$

\begin{tabular}{ll}
\multicolumn{1}{c}{ Stage } & \multicolumn{1}{c}{ Bed mobility } \\
\hline 1=Independence & Patient is able consistently to perform skill safely with no one present \\
2=Supervision & Patient requires someone within arm's reach as a precaution \\
3=Minimal assistance & Patient is able to complete majority of the activity without assistance \\
4=Moderate assistance & Patient is able to complete part of the activity without assistance \\
5=Maximal assistance & Patient is unable to assist in any part of the activity \\
\hline
\end{tabular}

Appendix 2. Content of the Ability for Basic Movement Scale (ABMS) ${ }^{6}$

\begin{tabular}{ll}
\hline \multicolumn{1}{c}{ Stage } & \multicolumn{1}{c}{ Sit up } \\
\hline 0=Prohibited from moving & $\begin{array}{c}\text { Patient prohibited from moving because of a medical problem, such as } \\
\text { unstable vital signs or complications }\end{array}$ \\
$\begin{array}{ll}\text { 1=Totally dependent } & \text { Movement with more than 75\% support by another person } \\
\text { 2=Partially dependent } & \text { Movement with 75\% or less support by another person }\end{array}$ \\
$\begin{array}{l}\text { 3=Independent in special environment } \\
\text { Independent in special environment: movement by holding a handrail or } \\
\text { edge of the bed or need to be watched by a supporter }\end{array}$ \\
\hline
\end{tabular}

